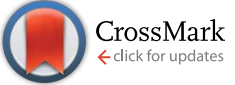

Cite this: RSC Adv., 2015, 5, 47466
Received 26th March 2015 Accepted 20th May 2015

DOI: $10.1039 / \mathrm{c} 5 \mathrm{ra05336f}$

www.rsc.org/advances

\section{Organogel formation via supramolecular assembly of oleic acid and sodium oleate}

\author{
Constantinos V. Nikiforidis, ${ }^{\text {*ab }}$ Elliot Paul Gilbert ${ }^{\star c}$ and Elke Scholten ${ }^{\text {ab }}$
}

To create materials with novel functionalities, the formation of gels within hydrophobic media has become popular. This is often accomplished through the assembly of low molecular weight organogelators into a variety of complex phases through intermolecular interactions. In the case of edible materials, the assembly of saturated fatty acids to form fat crystal networks is often used for structuring. Here, the first example of structuring with unsaturated fatty acids is reported, namely mixtures of oleic acid and sodium oleate, to structure edible lipid phases. Small-angle scattering demonstrates that the resultant structures, which vary with oleic acid and sodium oleate molar ratio, comprise either inverse micellar or lamellar phases, combined with the formation of crystalline space-filling networks. Network formation was found for filler concentrations above $10 \mathrm{wt} \%$. Rheological measurements show that gel strength depends on the ratio of oleic acid to sodium oleate, and is greater when only oleic acid is used. The addition of up to $1.5 \mathrm{wt} \%$ of water enhanced the strength of the organogels, probably through supplementary hydrogen bonding but, for concentrations greater than $2.0 \mathrm{wt} \%$, the assembly was inhibited leading to collapse of the gel.

\section{Introduction}

Organogels are a class of soft materials that can entrap large volumes of organic liquids in self-assembled networks, and can be used in numerous practical applications, ranging from photovoltaics, ${ }^{1}$ energy transfer, ${ }^{2}$ light harvesting ${ }^{2}$ and oil spill control, ${ }^{3}$ to bioactive compound release ${ }^{4}$ and appetite satiety. ${ }^{5}$ For edible applications, organogelators are often referred to as oleogelators. Although it is known that organogelation arises via the formation of various weak intermolecular interactions between the organogelator molecules to generate networks, ${ }^{6}$ there is still a lack of fundamental understanding considering the type of interactions that are necessary. The formation of these structures through supramolecular assembly is of particular interest because they could provide semi-solid bulk phases that are easily processable at low cost. The networks of organogels can range from assembly of surfactants in solution by physical interactions (e.g., micellization, lyotropism, and crystallization), ${ }^{7}$ also known as molecular organogels, to network formation of flexible polymers (e.g., swelling), known as polymer organogels. ${ }^{8}$ The currently known molecular organogels are at the interface between complex fluids ${ }^{9}$ and solids and, regardless of the nature of the structure, they are often thermoreversible quasi-solid materials. The group of molecular

${ }^{a}$ Top Institute Food \& Nutrition, P.O. Box 557, 6700AN Wageningen, The Netherlands. E-mail: costas.nikiforidis@wur.nl; Tel: +31 317485218

${ }^{b}$ Physics and Physical Chemistry of Foods, Wageningen University, P.O. Box 17, 6700AA, Wageningen, The Netherlands

${ }^{c}$ Bragg Institute, ANSTO, Locked Bag 2001, Kirrawee DC, NSW 2232, Australia. E-mail: elliot.gilbert@ansto.gov.au; Tel: +61297179470 organogels can be subcategorized according to the mode of the organogelator self-assembly into liquid crystals, ${ }^{10}$ platelet crystals, ${ }^{11}$ fibrillar networks ${ }^{12}$ and reverse worm-like micelles. ${ }^{\mathbf{1 3 1 4}}$

These different structures can be formed from amphiphilic compounds that have the ability to self-assemble into a variety of microstructures, including micellar phases and bilayers that, at high concentrations, can pack into different lyotropic mesophases of larger length scales, such as hexagonal, cubic or lamellar liquid crystalline phases ${ }^{\mathbf{1 5 , 1 6}}$ and, to a significant extent, predictable from the critical packing parameter. ${ }^{17}$ The formation of structures of larger length scales provide viscoelastic, or even gel-like, behaviour to the solution. These viscoelastic materials created through assembly of small gelators are often described in the literature as supramolecular organogels. ${ }^{18}$ The gelator molecules self-assemble into nanofibres (supramolecular polymers) of specific dimensions due to their geometrical packing with their entanglements creating a spacespanning three dimensional network that may entrap solvents in the nanospaces. The mechanism behind the formation of these structures is the array of monomer units that are linked through non-covalent bonds, such as van der Waals, hydrogen bonding, electrostatic interactions and $\pi-\pi$ or $\tau$-stacking. ${ }^{18,19}$

Overall, there are a limited number of biocompatible components known to structure lipids through the assembly of molecules. ${ }^{20-22}$ For example, gel formation was obtained with sodium carboxylates in organic solvents and ionic liquids ${ }^{23-25}$ and in different alcohols, such as ethanol and decanol, by sodium laurate. ${ }^{26}$ The gelation was explained by the fact that sodium laurate forms fibres in organic solvents (alcohols). ${ }^{27}$ The network formation of laurate salts was strongly influenced by 
the type of metal ion that was present. Sodium ions were shown to play an important role in the network formation and enhanced gel strength, whereas other metal ions, such as potassium, calcium and magnesium had limited effect on the gel formation. It was reported that the sodium ions could induce a transition from spherical micelles to cylindrical micelles, while other metal ions could not. ${ }^{26}$ Similar results have also been observed for other systems and it is noteworthy that, apart of the type of cations of the fatty salts, according to Daniel and Rajasekharan (2003), the length of the fatty chains can play an important role as well. Furthermore, it is already known that unsaturated fatty acids like oleic acid and their mixtures with fatty acid salts also form supramolecular assemblies in aqueous environments. ${ }^{28-34}$ Additionally, when no solvent is present, depending on the ratio between the molecules, micelles or lamellar phases are formed. ${ }^{35}$ However, little is known about the behaviour of these systems in hydrophobic environments.

Here, we use the current knowledge on molecular assembly and the effect of salts to induce network formation in hydrophobic environments. We have studied the assembly structures of oleic acid and sodium oleate in refined sunflower oil and directly compared behaviour for a sub-set of these formulations using a purely hydrophobic solvent, decane. Our findings show that, depending on their composition, they form different types of assembly structures. Their mutual assembly in hydrophobic media leads to the formation of inverse micelles or lamellar structures. In addition, crystalline regions were observed that develop into space-filling networks. A combination of techniques was used to reveal the mechanism of network formation, such as rheometry, differential scanning calorimetry, polarized microscopy, small-angle neutron scattering as well as dynamic light scattering.

\section{Experimental section}

\subsection{Materials}

Refined sunflower oil was purchased from commercial sources. Oleic acid (cis-9-octadecenoic acid) $\mathrm{C}_{18} \mathrm{H}_{34} \mathrm{O}_{2}$, sodium oleate (cis-9-octadecenoic acid sodium salt) $\mathrm{C}_{18} \mathrm{H}_{33} \mathrm{NaO}_{2}$ and all other chemicals were obtained from Sigma-Aldrich (Steinheim, Germany) at the highest degree of available purity. Sunflower oil was purified so only triglycerides are expected to be present. No analysis of the fatty acid composition was conducted but according to literature, the expected composition is: linoleic acid (polyunsaturated n-6): 48-74\%, oleic acid (monounsaturated n-9): 14-40\%, palmitic acid (saturated): $4-9 \%$ and stearic acid (saturated): 1-7\%. Hydrogenated decane (n-decane, purity $\geq 99 \mathrm{wt} \%$ ) and deuterated decane ( $n$-decane-d22, purity $\geq 99 \mathrm{wt} \%$ and deuteration level 99\%) was purchased from Sigma-Aldrich (Steinheim, Germany). Both solvents were used as received.

\subsection{Sample preparation}

All samples were prepared by mixing oleic acid and sodium oleate at different ratios $(1: 0,2: 1,1: 1,1: 2$, and $0: 1)$ in refined sunflower oil. The total concentration in sunflower oil was varied between 4 and $16 \mathrm{wt} \%$ and the amount of added water to each sample varied between 0 and $2 \mathrm{wt} \%$. All samples were first mixed with a high speed blender (Ultra Turrax, IKA, Germany) at $13.4 \mathrm{rpm} \mathrm{m^{-1 }}$ at room temperature for $30 \mathrm{~s}$. Immediately thereafter, the samples were heated at $80{ }^{\circ} \mathrm{C}$ for 20 min under stirring. The samples were then cooled down at room temperature and stored overnight at $5{ }^{\circ} \mathrm{C}$.

For small-angle neutron scattering experiments, selected samples were formulated using either deuterated or hydrogenated decane. Each sample contained $16 \mathrm{wt} \%$ of the filler (oleic acid : sodium oleate) at different ratios $(2: 1,1: 1,1: 2$ and $0: 1$ ). In addition, three samples of $1: 1$ oleic acid : sodium oleate were prepared (namely $1: 1 \mathrm{a}, 1: 1 \mathrm{~b}, 1: 1 \mathrm{c}$ ). All of the samples were dissolved in $1 \mathrm{wt} \%$ water and $83 \mathrm{wt} \%$ decane, but they differed with respect to the presence of hydrogenated or deuterated water or decane. For sample $1: 1 \mathrm{a}$, the filler was mixed with deuterium oxide and $n$-decane-d22 (DH); sample $1: 1 \mathrm{~b}$ was initially dissolved in $1 \mathrm{wt} \%$ water and $83 \mathrm{wt} \%$ $n$-decane-d22 (HD), and sample $1: 1 \mathrm{c}$ in $1 \mathrm{wt} \%$ deuterium oxide and $83 \mathrm{wt} \%$ decane (DD). Samples $1: 1(\mathrm{a})$, (b) and (c) thus differ in terms of the contrast of the solvents by deuteration: (a) both decane and water are deuterated and contrasted against hydrogenated oleate; (b) contrast between $n$-decane-d22 and all other hydrogenated components and (c) all components deuterated except water. Samples that were of sufficient low viscosity (liquid-like) were placed in Hellma cuvettes (2:1, $1: 1 \mathrm{a}, 1: 1 \mathrm{~b}, 1: 1 \mathrm{c} \& 1: 2$ ). The $0: 1$ sample i.e. sodium oleate only, was studied in a demountable cell. All cells had a path length of $1 \mathrm{~mm}$.

\subsection{Rheological measurements}

Rheological characterisation of organogels was performed with a stress-controlled Anton Paar rheometer (Anton Paar, Austria) with a MCR300 PP50-TEKP CF56 setup, using a $49.95 \mathrm{~mm}$ parallel plate configuration with $1.0 \mathrm{~mm}$ gap width. The temperature was regulated by a Paar Physica circulating water bath and a Peltier system (TEZ 150P/MCR) with an accuracy of $\pm 0.1^{\circ} \mathrm{C}$. The linear viscoelastic region was assessed by amplitude sweep experiments at a constant frequency of $1 \mathrm{~Hz}$. For all organogels, a constant deformation of $\gamma=0.01$ was used which was well within the linear viscoelastic region for all samples. Small deformation oscillatory measurements were performed over the frequency range of $0.1-10 \mathrm{~Hz}$ at $20{ }^{\circ} \mathrm{C}$ to obtain the storage $\left(G^{\prime}\right)$ and loss $\left(G^{\prime \prime}\right)$ moduli to describe the visco-elastic properties. When single $G^{\prime}$ values are presented, they were obtained at a frequency of $1 \mathrm{~Hz}$, in line with standard convention. ${ }^{36}$

\subsection{Microscopy}

The microstructure of the organogels was analysed by cross polarised light microscopy (Reichert-Jung Polyvar, Germany) with Plan $10 \times / 1 \times$ and Plan $40 \times / 1 \times$ objectives at NIZO Food Research, Ede. All gels where freshly prepared and kept at $20{ }^{\circ} \mathrm{C}$ for $2 \mathrm{~h}$. 


\subsection{Size analysis}

The size of the inverse surfactant structures was determined by dynamic light scattering (DLS ZetasizerNanozS, Malvern Instruments Ltd, UK). Small amounts ( $<$ CMC) of oleic acid and/ or sodium oleate were added to sunflower oil $(0.01 \mathrm{wt} \%)$ in ratios of $0: 1,1: 1$ and $1: 0$. All measurements were performed at $60{ }^{\circ} \mathrm{C}$ in a disposable capillary cell.

\subsection{Differential scanning calorimetry (DSC)}

Samples of mass 15-25 mg were weighed in an aluminium pan and sealed hermetically. As a reference, an empty hermetically sealed aluminium pan was used. Samples were first equilibrated for $4 \mathrm{~h}$ at $5{ }^{\circ} \mathrm{C}$ and, subsequently heated to $80{ }^{\circ} \mathrm{C}$ at $2{ }^{\circ} \mathrm{C}$ $\min ^{-1}$. All measurements were performed at NIZO Food Research, The Netherlands.

\subsection{Small angle neutron scattering}

SANS experiments were performed on the PACE instrument at the Laboratoire Léon Brillouin (CEA), Saclay, France; this instrument has been described previously. ${ }^{37}$ Three configurations were used: high $q(\mathrm{SSD}=2.5 \mathrm{~m}, \mathrm{SDD}=1 \mathrm{~m}, \lambda=5 \AA)$; medium $q(\mathrm{SSD}=5 \mathrm{~m}, \mathrm{SDD}=4.7 \mathrm{~m}, \lambda=5 \AA)$ and low $q(\mathrm{SSD}=$ $5 \mathrm{~m}, \mathrm{SDD}=4.7 \mathrm{~m}, \lambda=13 \AA$ ) with source aperture $=16 \mathrm{~mm}$ diameter and sample aperture $=7 \mathrm{~mm}$ diameter. A temperature control bath was used to assure a temperature of $21^{\circ} \mathrm{C}$. These configurations when merged provide a $q$ range of 0.0083 to $0.44 \AA^{-1}$ where $q$ is the magnitude of the scattering vector, defined by $q=4 \pi / \lambda \sin (\theta / 2), \lambda$ is the wavelength, here equal to $5 \AA$, with $10 \%$ resolution, and $\theta$ the scattering angle. Absolute scaling was accomplished using the scattering from $\mathrm{H}_{2} \mathrm{O}$ at high $q$.

Table 1 Neutron scattering length densities of the different components and solvents

\begin{tabular}{lll}
\hline Component & Physical density $/ \mathrm{g} \mathrm{cm}^{-3}$ & Neutron SLD $/ \times 10^{-6} \AA^{-2}$ \\
\hline$n$-Decane & 0.73 & -0.487 \\
$n$-Decane-d22 & 0.843 & 6.58 \\
$\mathrm{H}_{2} \mathrm{O}$ & 1.00 & -0.56 \\
$\mathrm{D}_{2} \mathrm{O}$ & 1.11 & 6.34 \\
Oleic acid & 0.895 & 0.0784 \\
Sodium oleate & 0.90 & 0.204
\end{tabular}

Table 2 Composition of the OA : SO mixtures. The total concentration of the mixtures was $16 \%$ (D: deuterated, $\mathrm{H}$ : hydrogenated)

\begin{tabular}{lll}
\hline $\begin{array}{l}\text { Oleic acid : sodium } \\
\text { oleate }\end{array}$ & \%Oleic acid & Neutron SLD $/ \times 10^{-6} \AA^{-2}$ \\
\hline $2: 1$ & $67 \%$ & 0.12 \\
$1: 1 \mathrm{DD}$ & $50 \%$ & 0.1412 \\
$1: 1 \mathrm{DH}$ & $50 \%$ & 0.1412 \\
$1: 1 \mathrm{HD}$ & $50 \%$ & 0.1412 \\
$1: 2$ & $33 \%$ & 0.162 \\
$0: 1$ & $0 \%$ & 0.204
\end{tabular}

The neutron scattering length densities for the components investigated are shown in Table 1 . Varying the composition of the OA : SO has a minimal effect on the overall neutron SLD varying from 0.1 to $0.2 \times 10^{-6}$ (Table 2), i.e. significantly less than that of the deuterated oil.

\section{Results and discussion}

\subsection{Gel formation}

Despite the diversity in chemical composition and physical properties of existing organogels, still the preferred and desired molecular architecture is not precisely known. Additionally, little is known about the relationship between the assembly behaviour of the gelator molecules and the mechanical properties of the gels. To evaluate the structure formation of different compositions of unsaturated fatty acids and fatty acid salts, oleic acid and sodium oleate (Fig. 1) have been mixed in different ratios in oil. When oleic acid was mixed with sunflower oil, even at concentrations above $20 \mathrm{wt} \%$, no significant changes in viscosity were observed, indicating that oleic acid is highly miscible with oil. This behaviour presumably results from the hydrophobic nature of oleic acid that has a hydrophilic-lipophilic balance (HLB) value around 1. This value indicates that oleic acid molecules are highly lipophilic and immiscible with water. On the other hand, the HLB value of sodium oleate is 18, which means that it is hydrophilic and can even act as a solubilizer or hydrotrope. ${ }^{38}$ When sodium oleate was added to sunflower oil, the system visibly increased in viscosity. This is a manifestation of the formation of large aggregates due to the low solubility of the sodium oleate in oil. Surprisingly, when oleic acid was combined with sodium oleate $(1: 1)$, a synergistic effect took place, leading to a very fine structured gel with a higher viscosity than the single-component systems (Fig. 2). The structure of these aggregates was further analysed with polarized light. As can be seen in Fig. 3, the gel structures resulting from (a) the mixture of oleic acid and sodium oleate, and from (b) sodium oleate only, have a completely different appearance. With only sodium oleate, large polydisperse, randomly distributed aggregates were formed but, in the case of sodium oleate combined with oleic acid, smaller crystals can be observed. As was expected, there was no evidence of crystal formation for oleic acid only (data not shown).

The type and the size of the aggregates depend on the assembly of both the sodium oleate and the oleic acid. Sodium

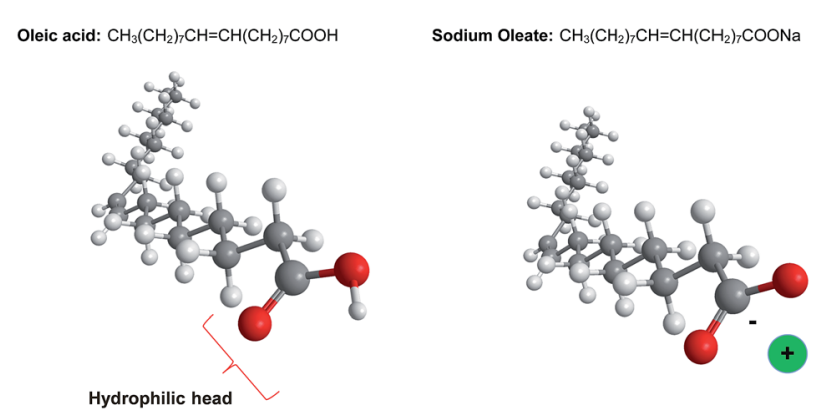

Fig. 1 Molecular structure of oleic acid and sodium oleate. 


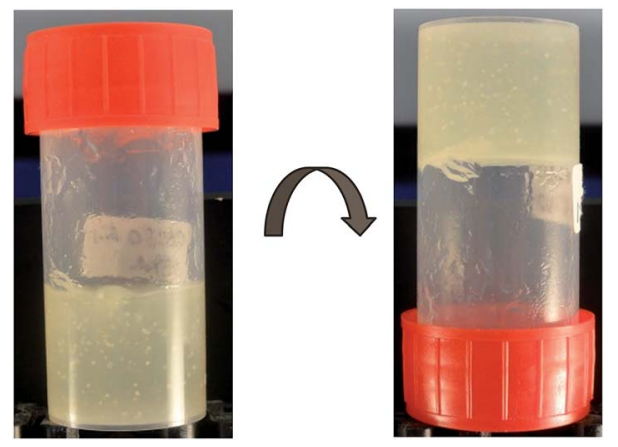

Fig. 2 Digital image of the non-pourable gel with $16 \mathrm{wt} \%$ of the oleic acid complex $(1: 1)$ in sunflower oil.
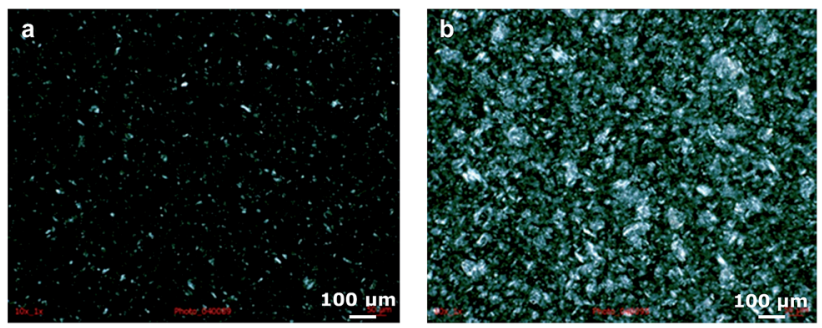

Fig. 3 Micrographs of organogels with $16.0 \mathrm{wt} \%$ of total structurant observed under crossed polarized light at $20{ }^{\circ} \mathrm{C}$. The oleic acid : sodium oleate ratio was $1: 1$ (a) and $0: 1$ (b).

oleate is an amphiphilic molecule, with a relatively high hydrophilicity due to the presence of the metal ion (Fig. 1). Therefore, hydrophilic interactions are more pronounced in a hydrophobic environment, which leads to extensive aggregation, as observed in Fig. 3. When oleic acid is added to the sodium oleate mixtures, the aggregation is diminished. The shape of the assembly is a result of the geometry of the molecules, along with the physical interactions between them.

\subsection{Dynamic light scattering}

To confirm the formation of the different assembly structures of the varying sodium oleate and oleic acid compositions, the sizes of the structural elements were measured with light scattering in a triglyceride environment. With oleic acid only, structures around $5 \mathrm{~nm}$ were identified. Assuming a fully extended tail conformation, oleic acid molecules would be expected to be $2.3 \mathrm{~nm}$ long but, due to the double bond, its size is expected to be closer to $2.0 \mathrm{~nm}\left(\mathrm{C}_{18} \mathrm{H}_{34} \mathrm{O}_{2}: 16\right.$ single $\mathrm{C}-\mathrm{C}$ bonds $(0.154 \mathrm{~nm})$, $1 \mathrm{C}=\mathrm{C}$ bond $(0.120 \mathrm{~nm}), 1$ paraffinic $\mathrm{C}-\mathrm{O}$ bond $(0.132 \mathrm{~nm})$ and $1 \mathrm{O}-\mathrm{H}$ bond $(0.097 \mathrm{~nm})$ ). A size of $5 \mathrm{~nm}$ would therefore indicate inverse micelle formation with a radius comparable to the size of the molecules. ${ }^{34}$ When only sodium oleate was added to sunflower oil, results from DLS experiments showed that large aggregates of sizes around $170 \mathrm{~nm}$ were formed, even after heating at $80^{\circ} \mathrm{C}$; this is consistent with the microscopy images, where large aggregates are observed. For different OA : SO mixtures, the size of the resulting structures were found to be around $25 \mathrm{~nm}$, much larger than the size of individual micelles. These results show that the presence of both oleic acid and sodium oleate leads to the formation of larger structures than for the presence of oleic acid only, and more extensive aggregation of sodium oleate is inhibited. This is, again, in agreement with the results found from polarized microscopy, and is a first indication that mutual packing between the two molecules indeed occurs. For a more detailed understanding of the different structures formed as a function of the composition (molar ratio) of the complexes, SANS experiments have been performed; these will be discussed later.

\subsection{Microstructure}

An integrated picture of the assembly mechanism and the synergistic effect of the molecules may be pursued by investigating the structures of different ratios between the two molecules. Several oleic acid : sodium oleate ratios that led to gel formation have been investigated, namely $3: 1,2: 1,1: 1,1: 2$, $1: 3$ and $0: 1$, at a total concentration of $16 \mathrm{wt} \%$ in sunflower oil. The formed gels were first observed with a polarized microscope, as is illustrated in Fig. 4 . It can be clearly seen that all gels have a different structure on a mesoscopic scale. For high concentrations of sodium oleate (Fig. $4 \mathrm{e}$ and f), a large degree of aggregation is seen, as discussed earlier. When more oleic acid is present (Fig. 4a-d), less aggregation with larger uniform areas is observed. In general, larger amounts of oleic acid led to larger areas of uniformity. According to the image in Fig. 4b, the formation of larger crystals was observed for a slight excess of oleic acid (ratio $2: 1$ ). Additionally, despite all gels being whitish-opaque, this particular sample was more transparent indicating the formation of structures below the wavelength of light. At a greater excess of oleic acid (Fig. 4a) the formed crystals were much smaller.

\subsection{Thermal behaviour}

The thermal behaviour of the samples (filler $16.0 \mathrm{wt} \%$, water $1.0 \mathrm{wt} \%$ ) was also investigated by DSC (Fig. 5) for a range of mixtures on the OA-rich side of the phase diagram as well as SO only. Samples i, ii and iii (the samples with a oleic acid : sodium oleate ratio of $3: 1,2: 1$ and $1: 1$ respectively) show that when oleic acid is present, phase transitions indeed occur, as a melting peak can be seen. However, these enthalpic changes do not occur in samples in the absence of oleic acid, (iv), over the observed thermal range. This may be understood from consideration of the reported phase diagram for $\mathrm{OA}$ : SO (without solvent) by Tandon and co-workers where the expected melting behaviour of OA is observed. ${ }^{29}$ No melting transition is predictably observed in the SO system as the melting point for SO occurs above $80{ }^{\circ} \mathrm{C}$. It is noted however that the expected trend of increasing transition temperature is not observed with increasing SO as reported in the un-solvated environment.

\subsection{Rheology}

The full mechanical spectrum of the formed organogel, with a total filler concentration of $16 \mathrm{wt} \%$ and an oleic acid to sodium 


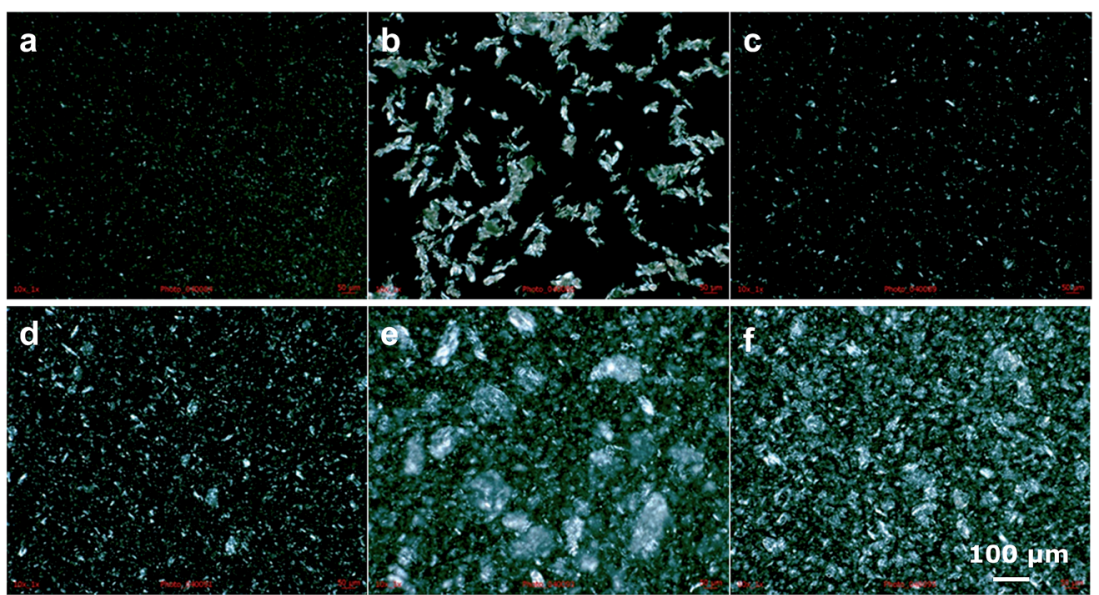

Fig. 4 Micrographs of organogels with $16.0 \mathrm{wt} \%$ of total structurant observed under crossed polarized light at $20^{\circ} \mathrm{C}$. The oleic acid : sodium oleate ratio was $3: 1$ (a), $2: 1$ (b), $1: 1$ (c), $1: 2$ (d), $1: 3$ (e) and $0: 1$ (f).

oleate ratio at $1: 1$, is given in Fig. 6 a. Due to the different network formation and aggregation behaviour, the gel strength of the resulting gels was also expected to be different. This is presented in Fig. 6b. The figure shows different ratios between the oleic acid and sodium oleate at the same total filler concentration (16 wt\%) in oil. Stronger gels were obtained for compositions in which both ingredients were present $(2: 1$, $1: 1$, and $1: 2$ ) and the gel strength of the three samples was comparable when obtained at a frequency of $1 \mathrm{~Hz}$. It is important to mention that the $\tan \delta$ values for the gels were around 0.15 for the ratios $2: 1,1: 1$, and $1: 2$, while it increased a little to 0.25 for the gels with ratios of $3: 1,1: 3$ and $0: 1$, indicating a slightly less strong network. Larger crystal structures, as seen in Fig. 4b for the 2:1 samples do not lead to a larger gel strength, so the gel formation is most likely more related to the connectivity between the crystals and the interactions that occur in the formed complexes.

To obtain information on the gel formation, the gel strength (storage modulus) of a $1: 1$ gel with different total filler

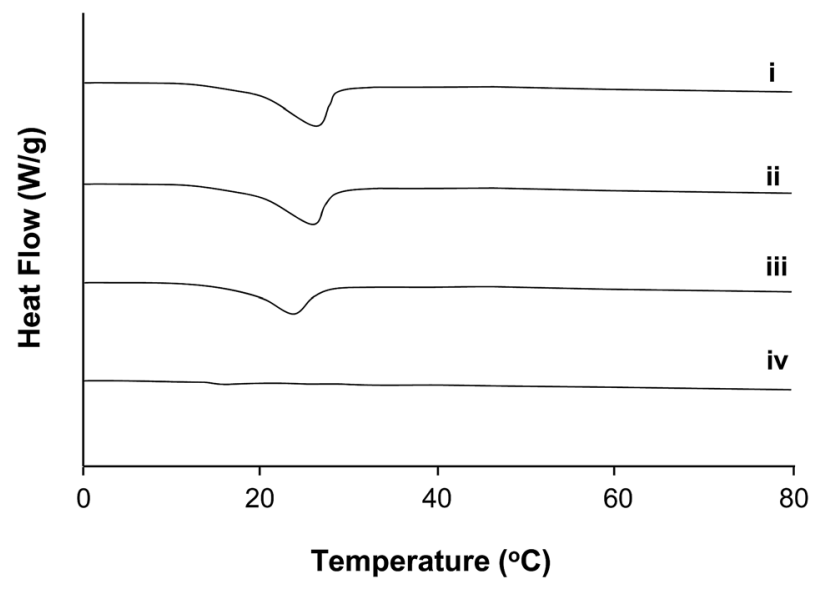

Fig. 5 Heat transitions of the crystalline network of organogels with 16.0 wt\% of total structurant. Lines i, ii, iii and iv, represent respectively oleic acid : sodium oleate ratios of $3: 1,2: 1,1: 1$ and $0: 1$. concentrations, denoted here as $c$, was investigated, as presented in Fig. 7 by the open symbols. The gel strength can be described as $G^{\prime} \sim c^{2.5}$, indicating more long range than short range interactions. ${ }^{39}$ For short range interactions, exponents closer to 3.3 are expected. Gel formation could be obtained even with 4 or $8 w$ wt of the complex, but these gels were not very stable after storage for several days. A filler concentration above
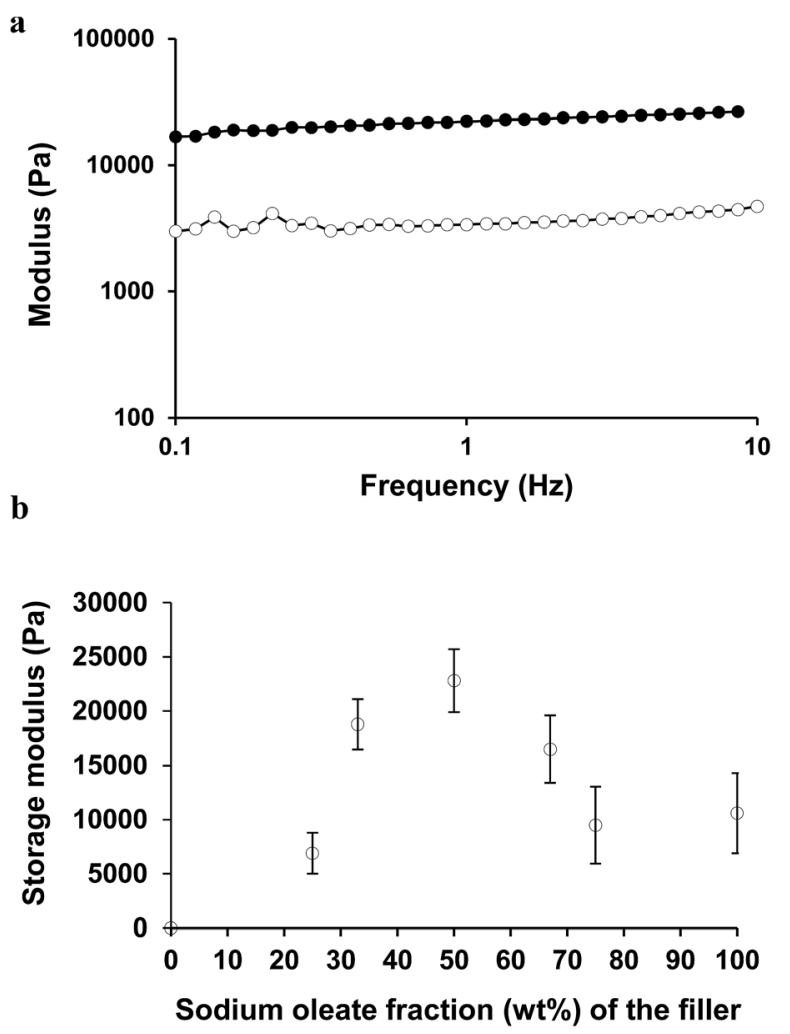

Fig. 6 Mechanical spectra $\left(20^{\circ} \mathrm{C}, \gamma=0.001\right)$ of an organogel with $16 \mathrm{wt} \%$ of total structurant in sunflower oil with a ratio of oleic acid to sodium oleate at $1: 1$ (a) and dependence of storage modulus $\left(20^{\circ} \mathrm{C}, \gamma\right.$ $=0.001)$ on different ratios between oleic acid and sodium oleate (16 wt\% of total structurant) (b). 


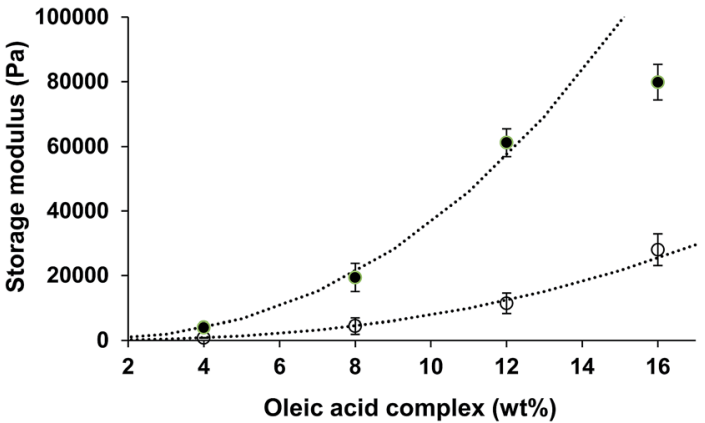

Fig. 7 Dependence of storage modulus $\left(20^{\circ} \mathrm{C}, \gamma=0.001\right)$ on different structurant concentration (oleic acid : sodium oleate, $1: 1$ ). The open symbols refer to samples without added water. The closed symbols refer to samples with $1.0 \mathrm{wt} \%$ water added. The lines were added to guide the eye and follow the best fit for $G^{\prime} \sim c^{\alpha}$, where $\alpha$ is the critical exponent.

$10 \mathrm{wt} \%$ would be appropriate for the formation of a solid-like system with sufficient storage stability. According to the literature, ${ }^{28,29}$ the oleic acid : sodium oleate complexes in an aqueous environment exhibit unique interactions through hydrogen bonding between the head group of the oleic acid and the carboxylate group of the salt, which is maximised for an equimolar ratio. ${ }^{40}$ The proposed mechanism is that the sodium atoms sit in the holes created by the non-hydrogen bonded oxygen atoms. ${ }^{25}$ In this way the complexes have both acid-like and carboxylate-like character that is different from either the parent soap or the parent acid.

As hydrogen bonding can clearly change the strength of the interactions, the effect of water addition has also been investigated. The addition of small amounts of water may increase the hydrogen bonding between the acid and the soap. As can be observed in the cross-polarized images in Fig. 8, the structure formation within the gels in comparison to the ones without water (Fig. 4) is very different. The changes are more profound for the gels with higher oleic acid concentration (Fig. 8a-c). Especially with a large excess of oleic acid (3:1 and $2: 1$ ratio), the crystals appear to have a much more needle-like shape. The more extensive crystal formation is a strong indication that the presence of water molecules enhances the interaction between oleic acid and sodium oleate molecules. The presence of small amounts of water increases the amount of hydrogen bonding between the polar heads of the molecules. The influence of water addition (0.5-2.0 wt\%) is shown as an increase in the gel strength (Fig. 8, closed symbols). For concentrations up to 16 $\mathrm{wt} \%$ of the filler concentration, the gel strength also increased with the concentration as $G^{\prime} \sim c^{2.5}$. The exponent of 2.5 is similar to that found in the case where no additional water present, indicating that the range of the interactions are similar for both systems. Most likely, without additional water present, the assembly formation was, besides (short range) van der Waals interactions, already dominated by (long range) hydrogen interactions, which is enhanced by the addition of water. For systems dominated mainly by van der Waals interactions, a higher exponent (closer to 3.3) for short interactions would have been expected. ${ }^{39}$ For higher concentrations, the gel strength deviates from this relationship, indicating that the strength of the interactions decreased.

Fig. 9 shows the gel strength as a function of the amount of water added and there is a limit in the amount of water that can be added. For water concentrations below $1.5 \mathrm{wt} \%$, the gels were always stronger than the ones without water, due to the increased hydrogen interactions, and exhibited similar rheological behaviour. When the water concentration exceeds $1.5 \mathrm{wt} \%$, the system is unable to accommodate the additional solvent within the assembled structures formed, and as a result, the system collapsed and phase separated. As the water has a higher affinity for the sodium oleate, the precipitant, a white solid-like material, was probably a mixture of sodium oleate and water, while the lipophilic oleic acid molecules probably remained in the supernatant.

\subsection{Nanostructure}

SANS is a valuable method to characterise the structure and selfassembly behavior of materials in the size range from one to several hundred nanometres and has found broad utilisation in

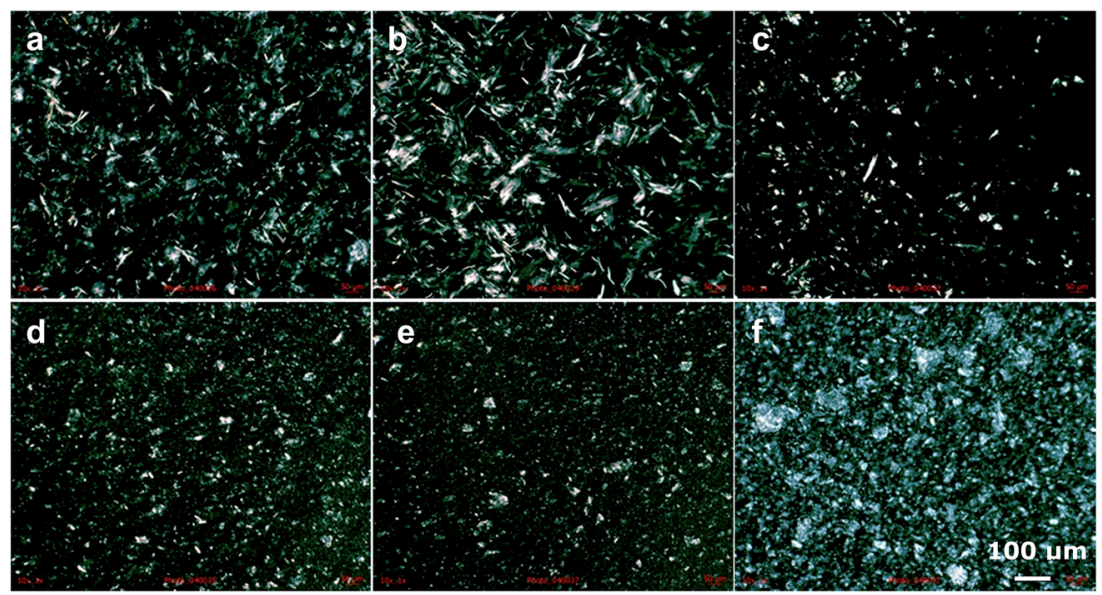

Fig. 8 Micrographs of organogels with $16.0 \mathrm{wt} \%$ of total structurant and $1.0 \mathrm{wt} \% \mathrm{H}_{2} \mathrm{O}$ observed under crossed polarized light at $20{ }^{\circ} \mathrm{C}$. The oleic acid : sodium oleate ratio was $3: 1$ (a), $2: 1$ (b), $1: 1$ (c), $1: 2$ (d), $1: 3$ (e) and $0: 1$ (f). 


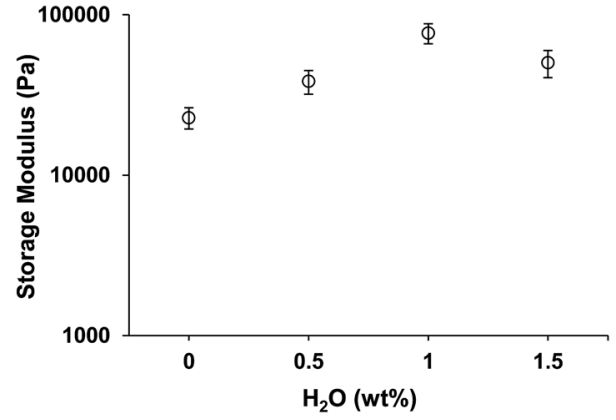

Fig. 9 Dependence of storage modulus $\left(20^{\circ} \mathrm{C}, \gamma=0.001\right)$ on different water concentration (16 wt\% oleic acid : sodium oleate, $1: 1$ ).

food-based systems. ${ }^{\mathbf{4 1}}$ The technique is most effective when employing selective deuteration; thus, to separately identify the influence of both oil and water on the resultant structures formed, the method requires use of deuterated molecules. As sunflower oil, which was used in all other experiments, is not available in a deuterated form, decane was selected to replace the oil as the hydrophobic phase, as it is readily obtainable in hydrogenated and deuterated form. It should be noted that the nature of decane, an unbranched hydrocarbon that exhibits only van der Waals interactions, is substantially different to sunflower oil both in amphiphilicity and purity (i.e. sunflower oil is itself a multi-component system). A direct comparison between the sunflower oil-based and decane-based systems is therefore beyond scope with this approach unless deuterated sunflower oil could be synthesised, for example, within a deuteration facility. ${ }^{\mathbf{4 2}}$ However, the results gained in decane are nonetheless informative and are valuable to interpret changes in the sunflower oil containing systems as will be demonstrated below.

Visual observations of the samples prior to conducting SANS studies (with decane as the continuous phase) are shown in Table 3. From these alone, the $2: 1$ (OA : SO) sample displays no evidence for structures on a length scale comparable to light. This is consistent with SANS data as will be described below. With increasing sodium oleate from $2: 1$ to $0: 1 \quad(0: 1$ is composed only of sodium oleate), the viscosity (gel formation) and opacity increases suggesting the formation of larger-scale structures. It is noted that deuteration appears to have an effect on the viscosity of the samples produced as observed for the three contrasts used in the equimolar system $(1: 1)$ although kinetic stabilisation effects cannot be excluded.

The SANS data for all systems is shown in Fig. 10. The SANS data from the $2: 1$ samples (Fig. 10A) exhibits a plateau at low $q$; this is consistent with visible transparency of the system. Guinier analysis yields a radius of gyration of approximately $7.3( \pm 0.4) \AA\left(q_{\max } R_{\mathrm{g}}<0.6\right)$. It is expected that inverse micelles are formed in such a formulation. Assuming a spherical morphology, the particle diameter, $D=2 \times(5 / 3)^{1 / 2}$ is ca. $19 \AA$ (close to that expected from full extended chains). Although this size is smaller than found with light scattering, it should be noted that the latter technique yields the hydrodynamic dimensions and is greater due to the solvation of the alkyl chains by the oil. To extract model dependent information, data were fitted to a polydisperse core-shell hard sphere interaction model (in which the core and shell have a fixed ratio) while constraining the volume fraction to $15.2 \%$ (based on known composition and physical density)* and the neutron scattering densities of the shell and solvent (based on literature values, Tables 1 and 2). Resultant fit parameters are shown in Table 4.

The SANS data from the $0: 1$ system (sodium oleate only, Fig. 10B), exhibits characteristic lamellar scattering with the presence of diffraction peaks at $0.149 ; 0.282$ and $0.429 \AA^{-1}$. The first reflection is consistent with a repeat distance of $43.5 \AA$; this value is in good agreement with that reported previously from the sodium oleate crystal structure $(c a .45 \AA){ }^{35}$ The scattering may be interpreted in terms of SO crystals in a hydrophobic matrix in which the water is presumably located around the ions. The scattering from a randomly oriented lamellar structure exhibits $q^{-2}$ scattering over a $q$ range from approximately $a^{-1}$ to $b^{-1}$, where $a$ and $b$ are the long and short dimensions of the structure, and transforms to $q^{-4}$ scattering at high $q$. Here $b^{-1}$ is at $c a$. $0.038 \AA^{-1}$ thus the minimum dimensions are $c a .165 \AA$ with the maximum dimensions of $\gg a^{-1}=0.008 \AA^{-1}(\gg 1250$ $\AA)$; the latter is consistent with the observed sample opacity and is comparable to the values obtained from light scattering. Assuming a lateral separation of $c a .4 \AA$, typical for alkyl chains, $165 \AA$ corresponds to approximately 40 sodium oleate chains side-by-side within the lamellar crystal structure. An estimate of the crystallite size, $L$, may be obtained by applying the Scherrer equation: $L=0.94 \lambda /(B \cos \theta)$, where $B$ is the angular width in radians at the full-width at half maximum of the diffraction peak and $2 \theta$ is the corresponding Bragg angle. All diffraction peaks in the $0: 1$ system have a similar width of $0.014 \AA^{-1}$; considering the influence of instrumental resolution to the peak broadening, this reduces to $0.011 \AA^{-1}$ yielding a crystallite size of approximately 470 Å.

The SANS data from the equimolar system $(1: 1)$ is shown in Fig. 10C. In this case, three compositions were investigated in

Table 3 Observation of different oleic acid : sodium oleate systems (D: deuterated, H: hydrogenated)

\begin{tabular}{|c|c|c|c|c|c|}
\hline $\mathrm{OA}: \mathrm{SO}$ & Oleic acid & Filler $(16 \% \mathrm{w} / \mathrm{w})$ & Oil $(83 \% \mathrm{w} / \mathrm{w})$ & Water $(1 \% \mathrm{w} / \mathrm{w})$ & Visual state prior to SANS \\
\hline $2: 1$ & $67 \%$ & $\mathrm{H}$ & $\mathrm{D}$ & $\mathrm{D}$ & Transparent liquid \\
\hline $1: 1 \mathrm{DD}$ & $50 \%$ & $\mathrm{H}$ & $\mathrm{D}$ & $\mathrm{D}$ & Opaque runny liquid \\
\hline $1: 1 \mathrm{DH}$ & $50 \%$ & $\mathrm{H}$ & $\mathrm{D}$ & $\mathrm{H}$ & Less runny liquid \\
\hline $1: 1 \mathrm{HD}$ & $50 \%$ & $\mathrm{H}$ & $\mathrm{H}$ & $\mathrm{D}$ & Even less runny liquid \\
\hline $1: 2$ & $33 \%$ & $\mathrm{H}$ & $\mathrm{D}$ & $\mathrm{D}$ & Runny gel \\
\hline $0: 1$ & $0 \%$ & $\mathrm{H}$ & $\mathrm{D}$ & $\mathrm{D}$ & Opaque gel \\
\hline
\end{tabular}



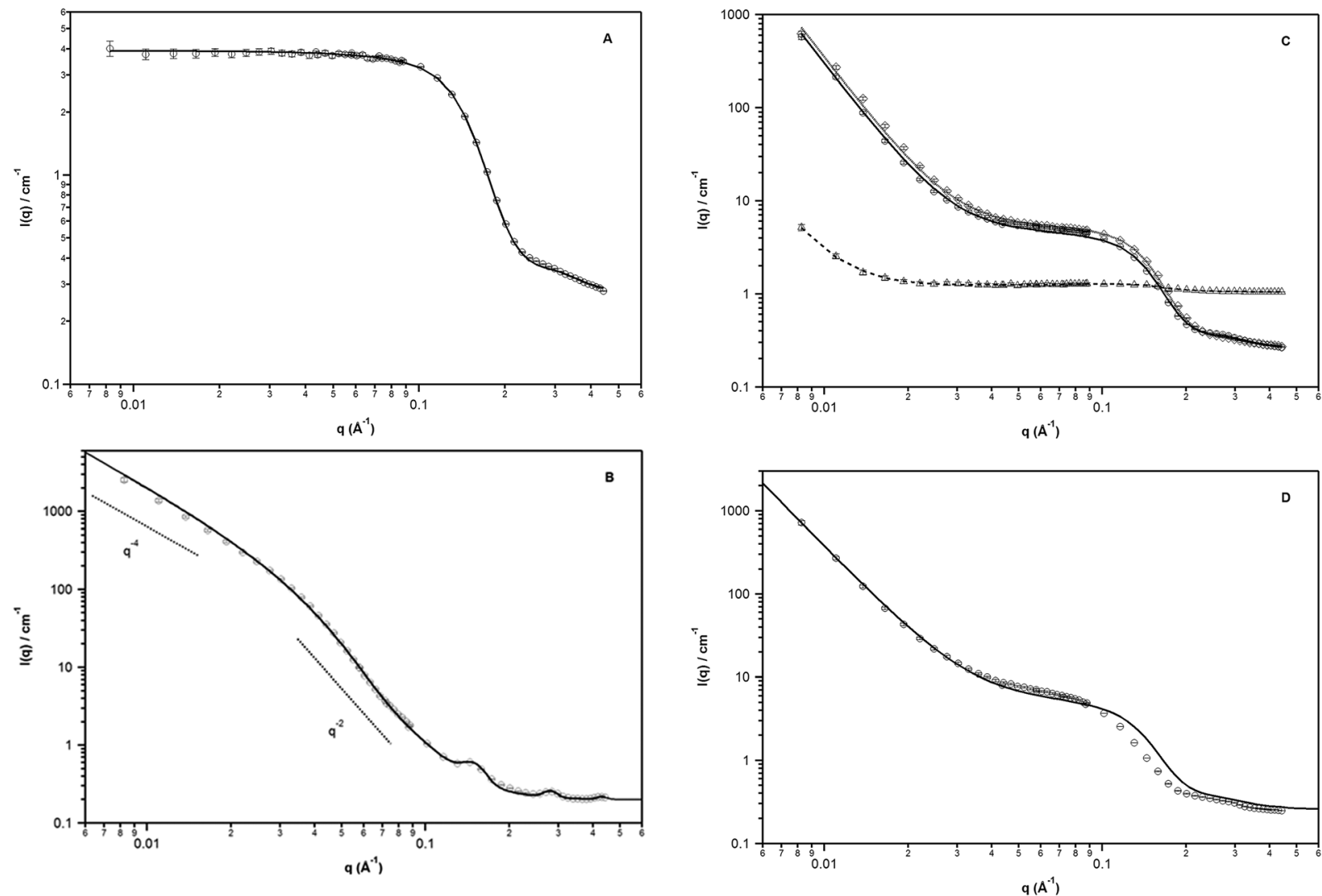

Fig. 10 SANS data for the different systems of oleic acid : sodium oleate (OA : SO) - open symbols - with associated model fits to the data solid lines - as described in the text; (A) 2 : 1 OA : SO including the associated fit to polydisperse core-shell hard sphere model; (B) 0 : 1 (sodium oleate only) and associated fit to lamellar model; (C) $1: 1$ system at three contrasts and associated fits to core-shell hard sphere inverse micelles and large scale structure (interfacial scattering) model (symbols: $1: 1 \mathrm{HD}$ presented as triangles; $1: 1 \mathrm{DD}$ presented as circles and $1: 1 \mathrm{DH}$ presented as diamonds) and (D) $1: 2$ system and associated 'best' fit to core-shell hard sphere micelles and large scale structure (interfacial scattering) model.

which either the water was deuterated, the decane was deuterated or both were deuterated. This enables three scattering patterns to be generated and to which a single model may be simultaneously fitted to all three data sets within which only the scattering length density is an unlinked refinable parameter. A model comprising a polydisperse core-shell model with hard sphere interaction (as used for the $2: 1$ system) combined with a power-law model to describe the low $q$ behaviour is shown to describe the scattering well. The associated fitting parameters are tabulated in Table 4 . The scattering may be ascribed to scattering from large-scale structures with a smooth and sharp interface (as evidenced by the approximately $q^{-4}$ scattering

Table 4 Fit parameters to SANS data based on model of inverse spherical micelles and large-scale structure formation. *The volume fraction may be estimated as follows: $0.16 /$ density $(0.9) /((0.16 / 0.9)+(0.83 /(0.73 \times 1.15493))+(0.01 \times 1))=$ ca. $15.2 \%$; this assumes that all material is in form of micelles and not free chains. The standard deviation in the last digit of the fitting parameter is shown in parentheses. (-) indicates the parameter is constrained (D: deuterated, $\mathrm{H}$ : hydrogenated)

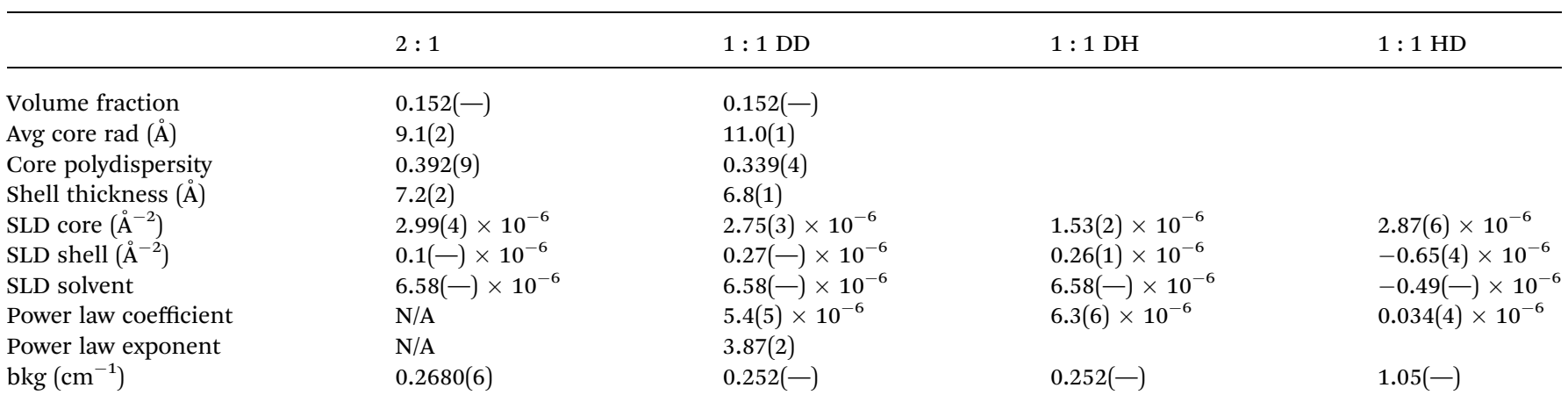


behaviour) in the presence of inverse micelles. It should be noted that the formation of large-scale structures is supported by the observed opacity from the samples for this composition. The core dimensions with the equimolar filler is also slightly larger compared to the $2: 1$ system (the former containing more sodium oleate) consistent with the presence of an increased amount of sodium oleate - which has the longer crystalline $d$ spacing - within the micellar phase.

The fits enable the extraction of information concerning the nature of the large-scale aggregated structure also. The power-law coefficients are $5.41( \pm 0.05), 6.3( \pm 0.6)$ and $0.034( \pm 0.004) \times$ $10^{-6}$ for the DD, DH and HD samples respectively. Thus, in the presence of hydrogenated solvent (the majority component of the system at $83 \% \mathrm{w} / \mathrm{w}$ ), there is very low contrast with respect to the aggregate. In the case of fully deuterated solvent (DD and DH), the power-law coefficient illustrates that the contrast is significantly greater relative to HD Using hydrogenated water (DH) results in a higher contrast compared to using deuterated water (DD) indicating that water is present within the large-scale aggregates. The latter is consistent with the findings above from rheology that indicates increased interactions with the addition of water.

SANS data from the 1:2 system (Fig. 10D) does not fit the same formalism as the other samples studied (Fig. 10A-C). However, based on the clear evidence of inverse micelle formation for the $1: 1$ systems and lamellar structures for the $0: 1$ system, it is likely that co-existence of the two (inverse micelles and lamellar structures) occurs. The combination of both structures is also observed as an intermediate macroscopic behaviour between a liquid and a gel. This co-existence can be noted from the low $q$ slope, which is closer to -3.3 , indicating an intermediate between lamellar $\left(q^{-2}\right)$ and large-scale structure formation $\left(q^{-4}\right)$. Furthermore, there is a small peak at $q$ at ca. $0.42 \AA^{-1}$ which is also observed for the $0: 1$ sample and a lower angle feature at ca. $0.27-0.28 \AA^{-1}$ can be seen, indicating presence of lamellar (crystal) structures. However, there is an insufficient number of contrast conditions, i.e. one compared to the 3 used for the $1: 1$ system, in the current case to verify this and it is not possible to exclude the existence of other potential structures (e.g. inverse cylindrical or hexagonal micelles). It is also noted that the assembly behaviour of OA and SO is complex and a variety of structures, e.g. vesicles, ${ }^{43}$ are observed in other solvents.

What appears to be clear is that with increasing sodium oleate concentration (i.e. from $2: 1$ to $0: 1$ ), the macroscopic characteristics trend towards gel formation, loss of transparency and large-scale structure formation. Since these large-scale structures appear to be crystalline in nature and predominantly composed of filler material (based on power-law coefficients), it is apparent that the addition of sodium oleate is causing the overall system to destabilise resulting in aggregation of the filler to form a spacefilling network. In addition, the observation that there is a trend from inverse micelles to lamellar structures upon increase in sodium oleate, and its associated change in interfacial curvature, is consistent with a change in packing parameter. This results from the association of the sodium ion to the oleate chain thus increasing the headgroup area, as discussed earlier.

\subsection{Molecular assembly}

The results show that the gel formation is a result of the mutual assembly of the components and the formation of large crystal structures. The mutual molecular assembly between oleic acid and sodium oleate was expected, since the presence of oleic acid can increase the solubility of sodium oleate in organic solvents, ${ }^{25,35}$ the complex formation and the inter-connectivity is likely to be the key factor in the formation of the different structures and the differences in the aggregate size of the crystals. Since the hydrophobic area (tail) of the two molecules is identical, the differences in assembly structures are dominated by the differences in their hydrophilic heads. In the case of oleic acid, the small hydrophilic head favours the formation of small inverse spherical micelles, with the hydrophobic tails present in the continuous oil phase. ${ }^{44}$ Inverse spherical micelles do not have the ability to overlap to form space spanning networks. For sodium oleate only, strong interactions lead to the formation of lamellar structures, and subsequently the appearance of larger crystals. When mixed, the involvement of sodium oleate (with a larger hydrophilic area) into the inverse micelles of oleic acid, induces a transition to lamellar crystalline structures that lead to network formation. The results from SANS and rheology indicate that complex formation is governed by hydrogen interactions. Additionally, the presence of water increases these attractive forces between the polar headgroups and thereby increases gel strength. Complementary hydrogen bonds between the hydrophilic heads of the two different molecules play an important role in the geometry of the assembly, ${ }^{45}$ since, in the hydrophobic solvent, the system seeks to minimise hydrophilic head group solvent interactions.

\section{Conclusions}

The assembly behaviour and subsequent gel formation of different compositions of an unsaturated fatty acid, oleic acid, and its salt, sodium oleate, in sunflower oil have been investigated. The presence of oleic acid alone did not provide any observable structural organization to the oil for gelation to occur. However, when oleic acid was mixed with sodium oleate, gel formation was induced. Different ratios of the two types of molecules gave a different appearance of the gels, indicating the presence of different microstructures. Polarized microscopy indicated that crystal formation occurred in selected samples but the spatial distribution differed with composition. SANS measurements indicate that in decane, in addition to crystal formation, for mixtures containing oleic acid, inverse micellar structures are also present. Participation of sodium oleate in the original inverse spherical micelles of oleic acid resulted in a change of the gelator geometry and assembly behaviour. For higher concentrations for sodium oleate, lamellar crystalline structures are formed. The mixtures of both components exhibit a phase transition in DSC experiments consistent with melting. The assembly is presumably driven to a large extent by hydrophilic interactions between the hydrophilic head groups, since the strength of the interactions increased by the addition of small amounts of water. For concentrations of water greater than 
$2 \%$, the assembly formation was inhibited. Apart from potential edible applications, the organogels with tuneable properties may have multiple applications in the pharmaceutical, chemical and oil technology industry.

\section{Acknowledgements}

The authors thank Sophie Combet, local contact on the PACE SANS instrument at the LLB.

\section{References}

1 W. Kubo, K. Murakoshi, T. Kitamura, S. Yoshida, M. Haruki, K. Hanabusa, H. Shirai, Y. Wada and S. Yanagida, J. Phys. Chem. B, 2001, 105, 12809-12815.

2 A. Ajayaghosh, V. K. Praveen and C. Vijayakumar, Chem. Soc. Rev., 2008, 37, 109-122.

3 S. Basak, J. Nanda and A. Banerjee, J. Mater. Chem., 2012, 22, 11658-11664.

4 B. Anand, S. S. Pisal, A. R. Paradkar and K. R. Mahadik, J. Sci. Ind. Res., 2001, 60, 311-318.

5 L. B. Sorensen, P. Moller, A. Flint, M. Martens and A. Raben, Int. J. Obes., 2003, 27, 1152-1166.

6 J. W. Steed, Chem. Commun., 2011, 47, 1379-1383.

7 H. Hoffmann, Adv. Colloid Interface Sci., 1990, 32, 123-150.

8 P. G. De Gennes, Scaling Concepts in Polymer Physics, Cornell University Press, Ithaca, NY, 1979.

9 W. M. Gelbart and A. BenShaul, J. Phys. Chem., 1996, 100, 13169-13189.

10 A. S. Sonin and N. A. Churochkina, Polym. Sci., Ser. A, 2013, 55, 353-384.

11 A. R. Hirst, D. K. Smith and J. P. Harrington, Chem.-Eur. J., 2005, 11, 6552-6559.

12 S. W. Wu, J. Gao, T. J. Emge and M. A. Rogers, Cryst. Growth Des., 2013, 13, 1360-1366.

13 M. Imai, K. Hashizaki, H. Taguchi, Y. Saito and S. Motohashi, J. Colloid Interface Sci., 2013, 403, 77-83.

14 C. V. Nikiforidis and E. Scholten, RSC Adv., 2014, 4, 24662473.

15 K. Trickett and J. Eastoe, Adv. Colloid Interface Sci., 2008, 144, 66-74.

$16 \mathrm{~J} . \quad$ N. Israelachvili, Intermolecular and Surface Forces, Academic Press, Elsevier, London, 2011.

17 J. N. Israelachvili, D. J. Mitchell and B. W. Ninham, J. Chem. Soc., Faraday Trans. 1, 1976, 72, 1525-1568.

18 M. Suzuki and K. Hanabusa, Chem. Soc. Rev., 2010, 39, 455463.

19 P. Dastidar, Chem. Soc. Rev., 2008, 37, 2699-2715.

20 E. D. Co and A. G. Marangoni, J. Am. Oil Chem. Soc., 2012, 89, 749-780.
21 S. Sahoo, N. Kumar, C. Bhattacharya, S. S. Sagiri, K. Jain, K. Pal, S. S. Ray and B. Nayak, Des. Monomers Polym., 2011, 14, 95-108.

22 J. Daniel and R. Rajasekharan, J. Am. Oil Chem. Soc., 2003, 80, 417-421.

23 W. Q. Jiang, J. C. Hao and Z. H. Wu, Langmuir, 2008, 24, 3150-3156.

24 J. M. Liang, Y. Ma, Y. Zheng, H. T. Davis, H. T. Chang, D. Binder, S. Abbas and F. L. Hsu, Langmuir, 2001, 17, 6447-6454.

25 R. Guo and Q. H. Fu, Chin. J. Chem., 2000, 18, 13-17.

26 D. Wang and J. C. Hao, Langmuir, 2011, 27, 1713-1717.

27 Z. W. Yuan, W. J. Lu, W. M. Liu and J. C. Hao, Soft Matter, 2008, 4, 1639-1644.

28 M. L. Lynch, Curr. Opin. Colloid Interface Sci., 1997, 2, 495500.

29 P. Tandon, S. Raudenkolb, R. H. H. Neubert, W. Rettig and S. Wartewig, Chem. Phys. Lipids, 2001, 109, 37-45.

30 M. Heppenstall-Butler and M. F. Butler, Langmuir, 2003, 19, 10061-10072.

31 J. M. Gebicki and M. Hicks, Nature, 1973, 243, 232-234.

32 W. R. Hargreaves and D. W. Deamer, Biochemistry, 1978, 17, 3759-3768.

33 B. Dejanovic, V. Noethig-Laslo, M. Sentjurc and P. Walde, Chem. Phys. Lipids, 2011, 164, 83-88.

34 K. Morigaki and P. Walde, Curr. Opin. Colloid Interface Sci., 2007, 12, 75-80.

35 P. Tandon, S. Raudenkolb, R. H. H. Neubert, W. Rettig and S. Wartewig, Chem. Phys. Lipids, 2001, 109, 37-45.

36 A. R. Patel, M. Babaahmadi, A. Lesaffer and K. Dewettinck, J. Agric. Food Chem., 2015, 63, 4862-4869.

37 C. Drappier, H. Oliveira, O. Sandre, E. Ibarboure, S. Combet, E. Garanger and S. Lecommandoux, Faraday Discuss., 2013, 166, 83-100.

38 S. E. Friberg, Curr. Opin. Colloid Interface Sci., 1997, 2, 490494.

39 V. Prasad, V. Trappe, A. D. Dinsmore, P. N. Segre, L. Cipelletti and D. A. Weitz, Faraday Discuss., 2003, 123, 1-12.

40 S. Han, Chem. Phys. Lipids, 2013, 175, 79-83.

41 A. Lopez-Rubio and E. P. Gilbert, Trends Food Sci. Technol., 2009, 20, 576-586.

42 http://www.ansto.gov.au/ResearchHub/Bragg/Facilities/National DeuterationFacility/.

43 M. Delample, F. Jerome, J. Barrault and J. P. Douliez, Green Chem., 2011, 13, 64-68.

44 C. V. Nikiforidis, in Edible Nanostructures: A Bottom-up Approach, ed. A. G. M. D. Pink, RSC, Cambridge, UK, 2015.

45 J. M. Lehn, M. Mascal, A. Decian and J. Fischer, J. Chem. Soc., Chem. Commun., 1990, 479-481. 